\title{
First cross section measurement of neutrino charged current interactions in the iron ECC
}

\section{H. Oshima*, H. Shibuya, S. Ogawa, T. Matsuo, Y. Morimoto, Y. Kosakai, K. Mizuno, H. Takagi for the NINJA collaboration}

Toho University, Department of physics, Funabashi, Chiba 274-8510, Japan

E-mail: 74160010@st.toho-u.jp

\begin{abstract}
Emulsion Cloud Chamber(ECC) with $65 \mathrm{~kg}$ iron target was exposed to the neutrino beam at JPARC neutrino beamline in 2016. It is capable of detecting slow protons as low as $200 \mathrm{MeV} / \mathrm{c}$. In this document, we use the data set corresponding to $0.40 \times 10^{20}$ protons on target. A total of 194 neutrino-iron charged current interactions were successfully reconstructed. Emission angles and momenta of muon and protons in the final state of each event were measured and compared with those of a Monte Carlo simulation. This is the first measurement using the iron ECC in this energy region.
\end{abstract}

The 21st international workshop on neutrinos from accelerators (NuFact2019)

August 26 - August 31, 2019

Daegu, Korea

${ }^{*}$ Speaker. 


\section{Introduction}

Overall view of neutrino-nucleus interactions around $1 \mathrm{GeV}$ is not clear due to final state interactions. There is a discrepancy between the measurements of the neutrino charged current quasielastic scattering cross section for around $1 \mathrm{GeV}$ and above $10 \mathrm{GeV}$ by several experiments [1]. In this energy region, nuclear effects and multi-nucleon scattering that have been known by electron scattering are major uncertainties for neutrino-nucleus interactions. We introduce an emulsionbased detector, a set of sub-micron resolution Emulsion Cloud Chambers(ECCs) with wide angle acceptance, as a neutrino detector. We can measure charged hadron final states with low energy threshold. In this document, emission angles and momenta of muon and protons in the final state of each event were measured and compared with those of a Monte Carlo simulation. These measurements are useful for cross section measurements.

\section{The NINJA experiment}

The Neutrino Interaction research with Nuclear emulsion and J-PARC Accelerator (NINJA) experiment uses an emulsion-based detector to study neutrino-nucleus interactions in the energy range of hundreds of $\mathrm{MeV}$ to a few $\mathrm{GeV}$. Since 2014 we have carried out a series of test experiments to evaluate our detector performance. In this document, we report results of the 2016 NINJA iron target run. Detector structure of the NINJA iron target run in 2016 is shown in Fig. 1) The detector consists of ECCs [2], a shifter(emulsion multi-stage shifter) [3 and INGRID(Interactive Neutrino GRID) [4]. Each ECC is a tracking detector made of emulsion films interleaved with iron plates(SUS304). Size of an emulsion film is $25 \mathrm{~cm} \times 25 \mathrm{~cm}$ and its thickness is $300 \mu \mathrm{m}$. The iron plate is of the same of emulsion film with thickness of $500 \mu \mathrm{m}$. Nuclear emulsion is well suited for precise measurement of positions and angles of interacting particles due to its sub-micron spatial resolution. Fig. 2 shows the image of a neutrino interaction in an emulsion layer taken by a microscope system, FTS [5, 6]. We can get fine information of neutrino interactions using ECC. Momentum of a charged particle is measured by using the Range - energy relation for a short track or measurement of Multiple Coulomb Scattering by the coordinate method[7] or the angular method[8]. Shifter is a time stamper consisted of emulsion films. That gives timing information of each ECC track. After the time stamping, ECC tracks and INGRID tracks are matched using timing information. INGRID is a near detector for the T2K experiment. That is used as a muon range detector.

\section{Expected neutrino beam flux and interactions}

Fig. 3-(a) shows the predicted neutrino flux at the detectors by JNUBEAM[9]. JNUBEAM is GEANT3[10]-base simulator for neutrino flux prediction. The average energy of the neutrino beam is $1.49 \mathrm{GeV}$ and the peak energy is $0.90 \mathrm{GeV}$. Fig. 3-(b) shows the expected neutrino-iron CC interactions in the ECCs by NEUT[11,12]. NEUT is a neutrino event generator and is used by Super-Kamiokande, T2K and the various experiments. Purity of the neutrino-iron CC interaction is $98.6 \%$. 

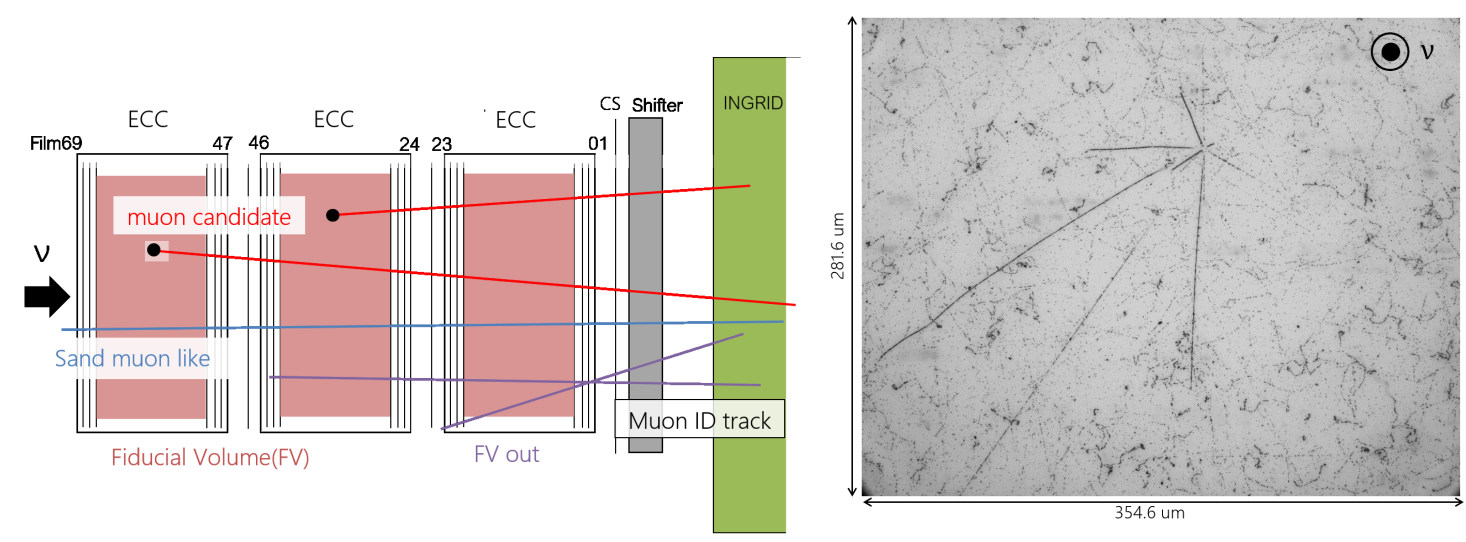

Figure 1: Detector structure and muon ID scheme of Figure 2: Image of a neutrino interaction in an emulNINJA iron target run in 2016. sion layer taken by FTS. The Black tracks are due to heavily ionizing particles emitted from the vertex.

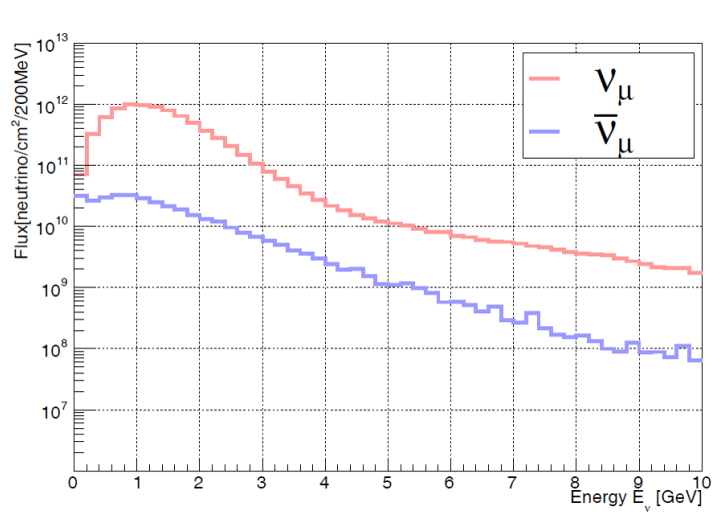

(a) Neutrino beam flux at the detectors.

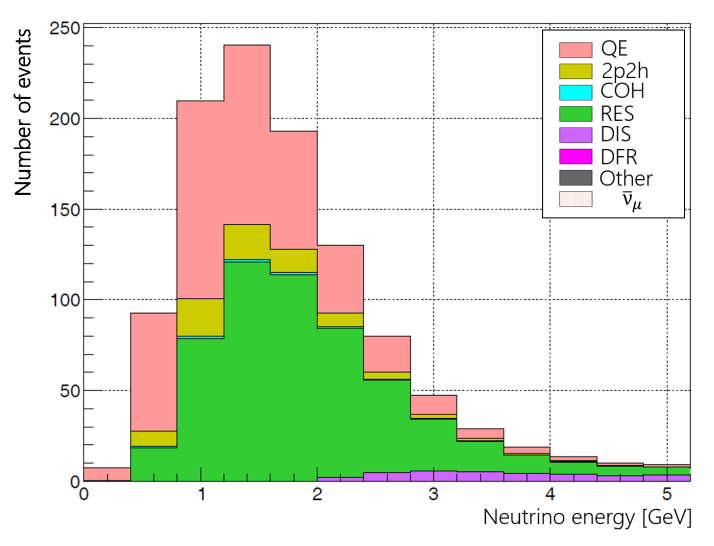

(b) Neutrino-iron CC interaction energy distribution.

Figure 3: Expected neutirno beam flux and CC interaction energy distribution.

\section{Event selection}

Data were taken from the exposure of iron target ECCs to the neutrino and the anti neutrino beam corresponding to integrated POT $0.40 \times 10^{20}$ and $3.53 \times 10^{20}$, respectively. Neutrino charged current interaction candidates are identified by the scanback method. The scanback method is a neutrino interaction identification method using an ECC and an electronic tracking detector. In this method, by tracing back to the neutrino interaction vertex in the ECC from INGRID via the shifter, CC interaction candidates are selected. We find 47,901 muon ID tracks which were selected by INGRID, however, most of these muon ID tracks are fiducial-volume-out tracks of ECCs or charged particle tracks from neutrino interactions occurred at the wall of the detector hall. Of those muon ID tracks, 1,318 tracks reached neutrino or anti-neutrino CC interaction vertices in ECCs. After selecting the neutrino beam timing, we obtained 194 neutrino-iron CC events in the neutrino beam mode. In this document, we study these 194 CC interactions. 


\section{Results}

\subsection{Muon kinematics}

Muon tracks with $\left|\tan \theta_{x}\right| \leq 1.7$ and $\left|\tan \theta_{y}\right| \leq 1.7$ are used in the following analysis. The positions $(\mathrm{x}, \mathrm{y})$ and slopes $\left(\tan \theta_{x}, \tan \theta_{y}\right)$ of the muon tracks are measured with respect to the microscope's coordinate system. The perpendicular of the emulsion film is defined as the z-axis of the microscope's coordinate system. Muon ID required the number of INGRID iron layers greater than or equal to 2 for tracking. That corresponds to muon momentum threshold about $300 \mathrm{MeV} / \mathrm{c}$. Fig. 45(a) shows the emission angle distribution of muons emitted from neutrino-iron interactions and Fig. 4-(b) shows the momentum. Angular distribution is limited to the forward direction because the muon ID is performed by matching the ECC, the shifter and INGRID. Data and Monte Carlo simulation agree well.

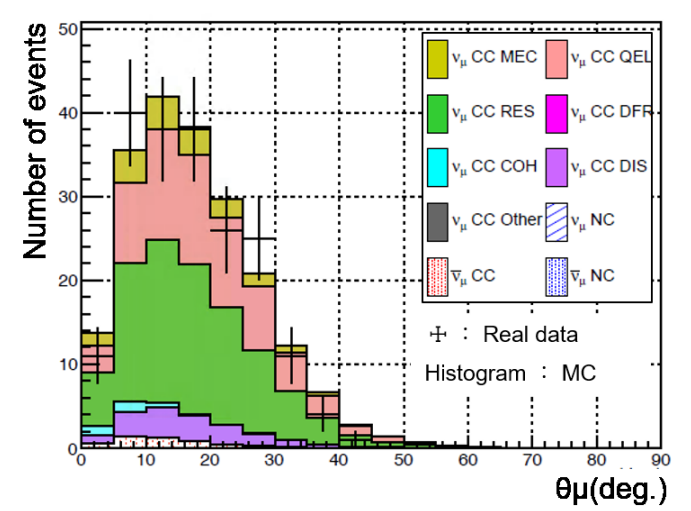

(a) Emission angle.

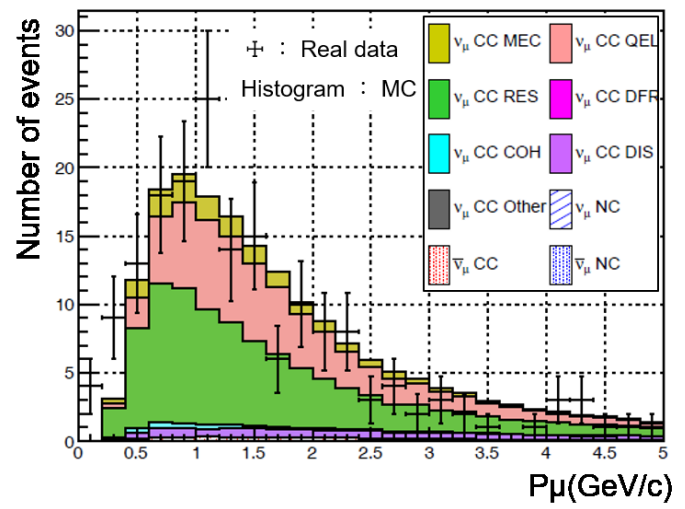

(b) Momentum.

Figure 4: Measurements of emission angle and momentum for muon.

\subsection{Proton kinematics}

Proton tracks with $\left|\tan \theta_{x}\right| \leq 1.7$ and $\left|\tan \theta_{y}\right| \leq 1.7$ and 2 or more emulsion layers are used for analysis. This corresponds to momentum threshold about $200 \mathrm{MeV} / \mathrm{c}$ for proton. Proton ID is performed using the likelihood ratio defined by their momentum and volume pulse height which is a measure of $\mathrm{dE} / \mathrm{dx}$. Fig. 5-(a) shows the emission angle distribution of protons emitted from neutrino-iron interactions and Fig. 5-(b) shows the momentum of these protons. Data is in good agreement with Monte Carlo simulation. Low momentum protons down to about $200 \mathrm{MeV} / \mathrm{c}$ were successfully detected.

\section{Summary}

Study of neutrino-nucleus interactions in sub-multi GeV energy region is very important for current and future neutrino oscillation experiments. A $65 \mathrm{~kg}$ iron ECC target was exposed to the neutrino beam with a mean energy of $1.5 \mathrm{GeV}$ at J-PARC in 2016 . From this exposure of $0.40 \times 10^{20}$ POT, 194 neutrino-iron CC interactions were identified. We can detect charged hadrons in the final 


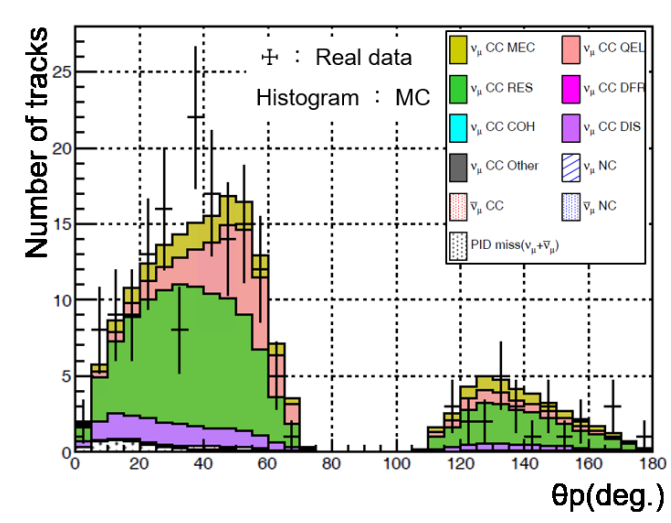

(a) Emission angle.

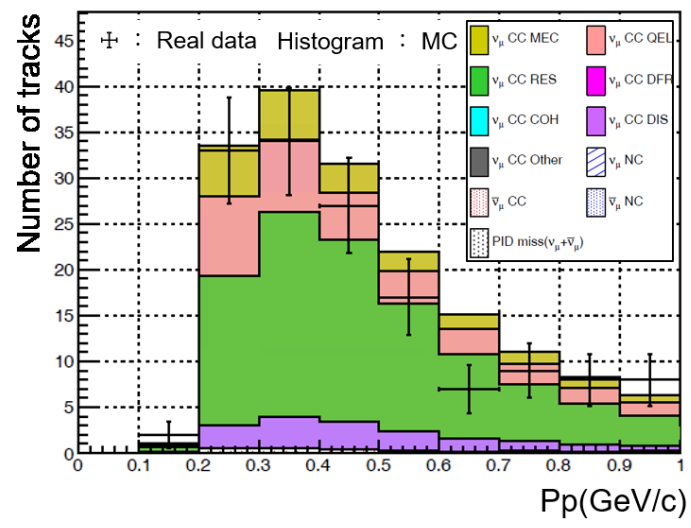

(b) Momentum.

Figure 5: Measurements of emission angle and momentum for protons.

state with low momentum thresholds: $200 \mathrm{MeV} / \mathrm{c}$ for protons and $50 \mathrm{MeV} / \mathrm{c}$ for charged pions. We compare the measurement of emission angles and momenta for muon and protons from neutrino interaction and those of the Monte Caro simulation.

\section{References}

[1] A. A. AGUILAR-AREVALO et al., Phys. Rev. D 81, 092005 (2010).

[2] T. Fukuda et al., Prog. Theor. Exp. Phys. $063 \mathrm{C} 02$ (2017).

[3] K. Yamada et al., Prog. Theor. Exp. Phys. 063H02 (2017).

[4] K. Abe et al., Nucl. Instrum. Meth. Phys. Res. A 694 (2012) 211-223.

[5] T. Fukuda et al., JINST, 8, P01023 (2013).

[6] T. Fukuda et al., JINST, 9, P12017 (2014).

[7] K. Kodama et al., Nucl. Instr. and Meth. A 574 (2007) 192-198.

[8] N. Agafonova et al., New J. Phys. 14 (2012) 013026.

[9] K. Abe et al., Phys. Rev. D 87, 012001 (2013) (2016).

[10] R. Brun, F. Carminati, and S. Giani, Report No. CERNW5013 (1994).

[11] Y. Hayato, Nucl. Phys. B Proc. Suppl. 112, 171 (2002).

[12] Y. Hayato, Acta Phys. Pol. B 40, 2477 (2009). 